\section{Deponiesanierung und Bau einer Reststoffdeponie}

\author{
Raymond Vouillamoz*
}

\section{Einleitung}

Die Walliser Werke der Lonza $A G$ betreiben seit 1923 in Gamsenried, auf dem Gebiet der Gemeinde Brig-Glis, eine Deponie für Produktionsrückstände.

Talaufwärts wird die Deponie durch den Schuttkegel der Gamsa begrenzt, der sich über rund zwei Drittel der Talebene er- dehnung etwa 24 ha und wurde inzwischen durch Verkehrsbauten wie die Kantonsstrasse auf rund 18 ha reduziert.

\section{Ursache der Verschmutzung}

Bis 1962 gelangte einzig Kalkhydrat aus der Acetylengewinnung zur Ablagerung. Dieses Kalkhydrat bestand aus einem Schlamm von grosser Reinheit, der vom Werk zur Deponie gepumpt und anschliessend laguniert wurde $(4 \mathrm{~km})$. Die Mächtigkeit der Kalkhydratschicht erreicht örtlich bis zu $15 \mathrm{~m}$. Dieses Kalkhydrat wird heute genutzt und als Neutralisationsmittel (Kalkmilch) von Abwässern eingesetzt. Auf den insgesamt 24 ha Deponiegelände wurden etwa 1,5 Mio. $\mathrm{m}^{3}$ schlammförmige Rückstände deponiert.

Da man aufgrund der damals vorhandenen Kenntnisse und Beobachtungen annahm, die verfestigten Schlammschichten seien praktisch wasserundurchlässig, wurden zwischen 1963 und 1978 auch organische Produktionsrückstände auf die Deponie gebracht.

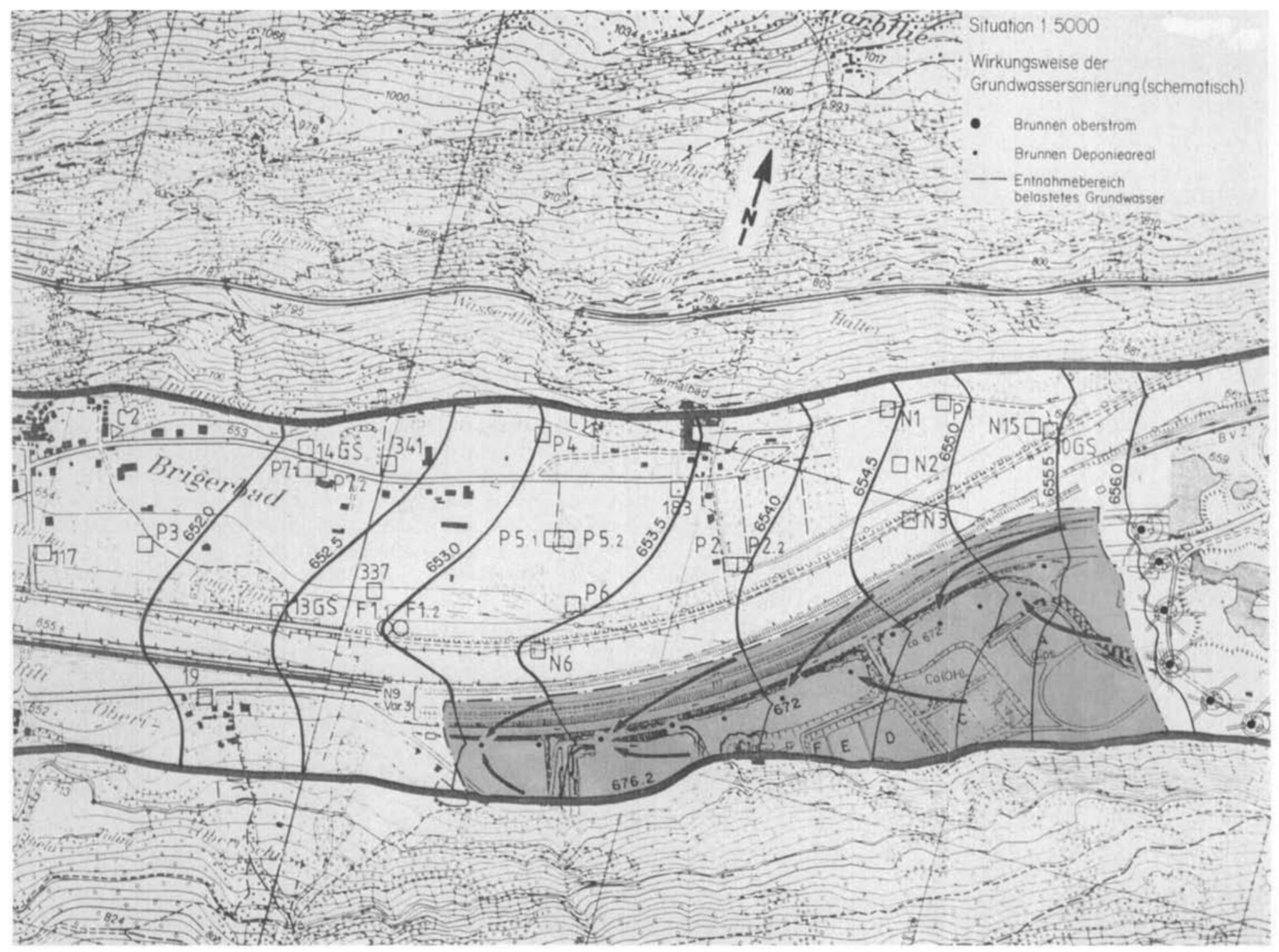




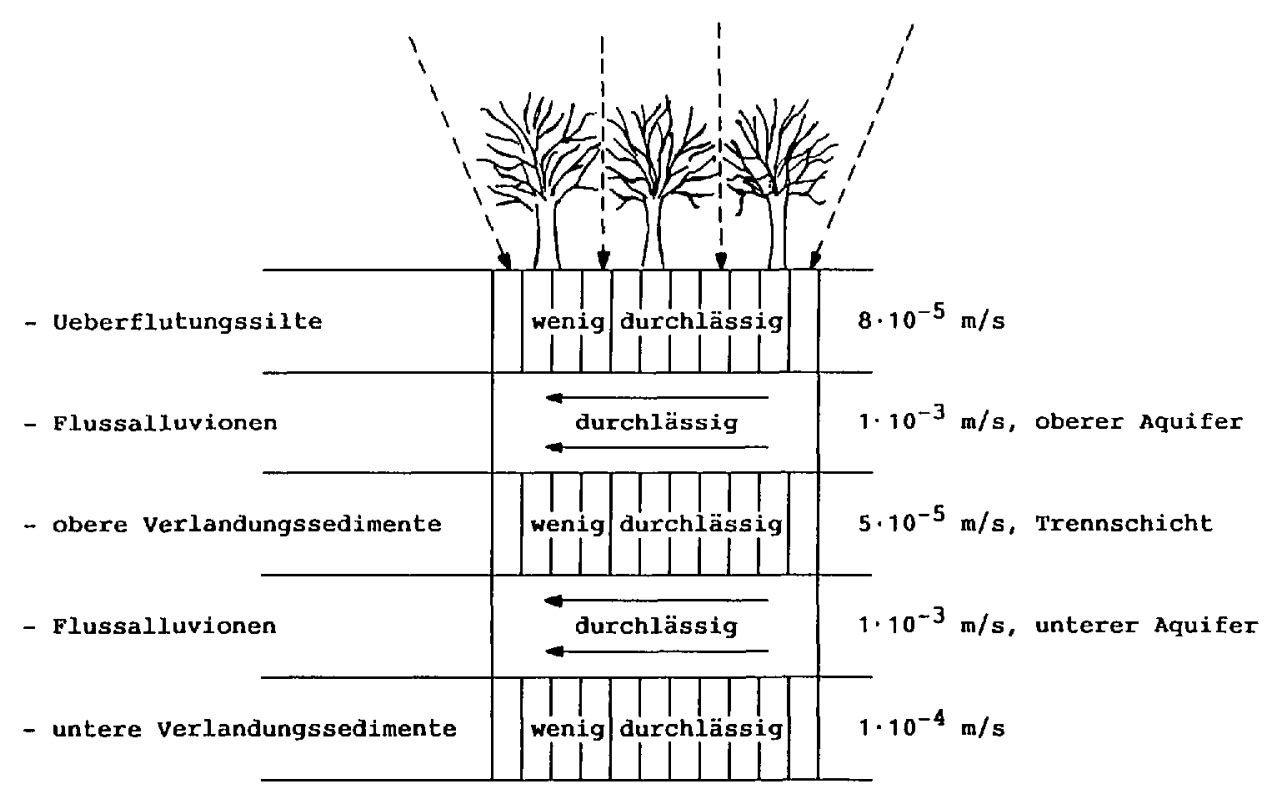

Fig. 2

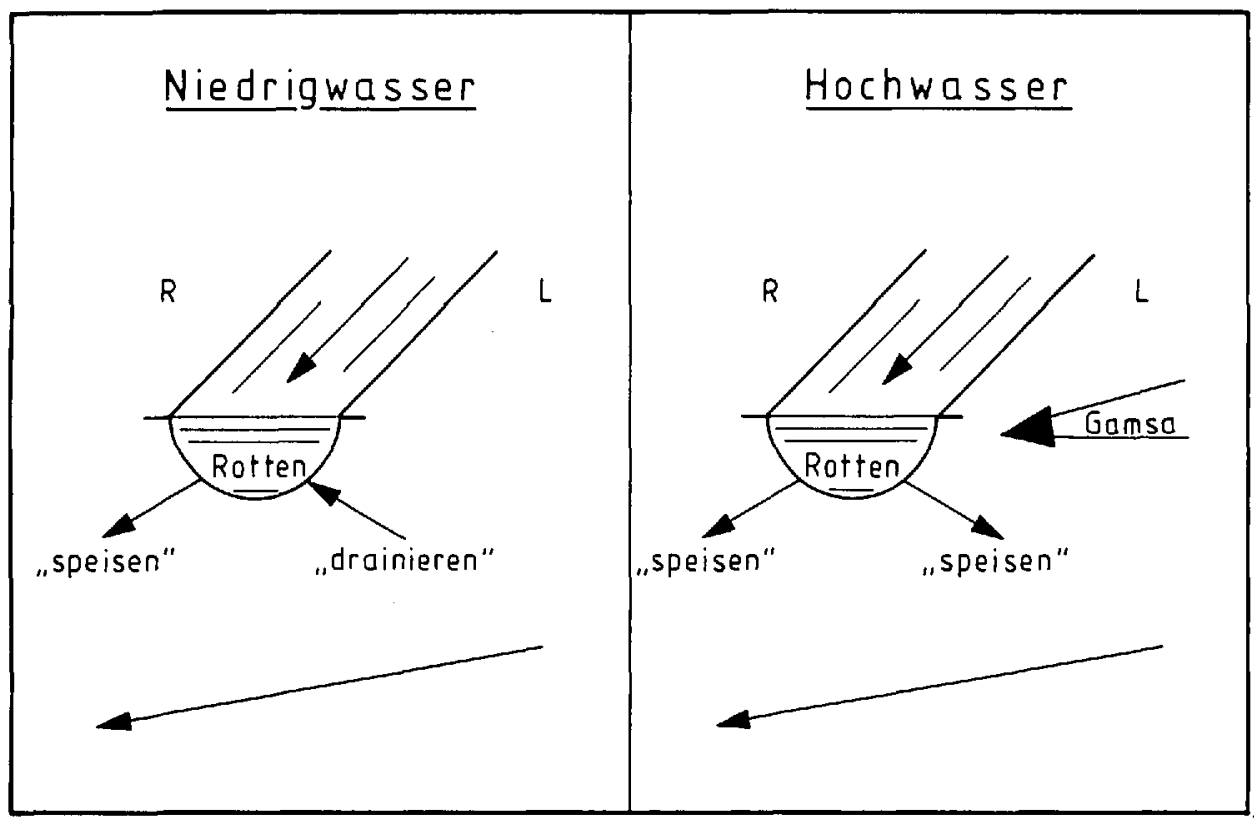

Fig. 3. Niedrig- und Hochwasserbedingungen

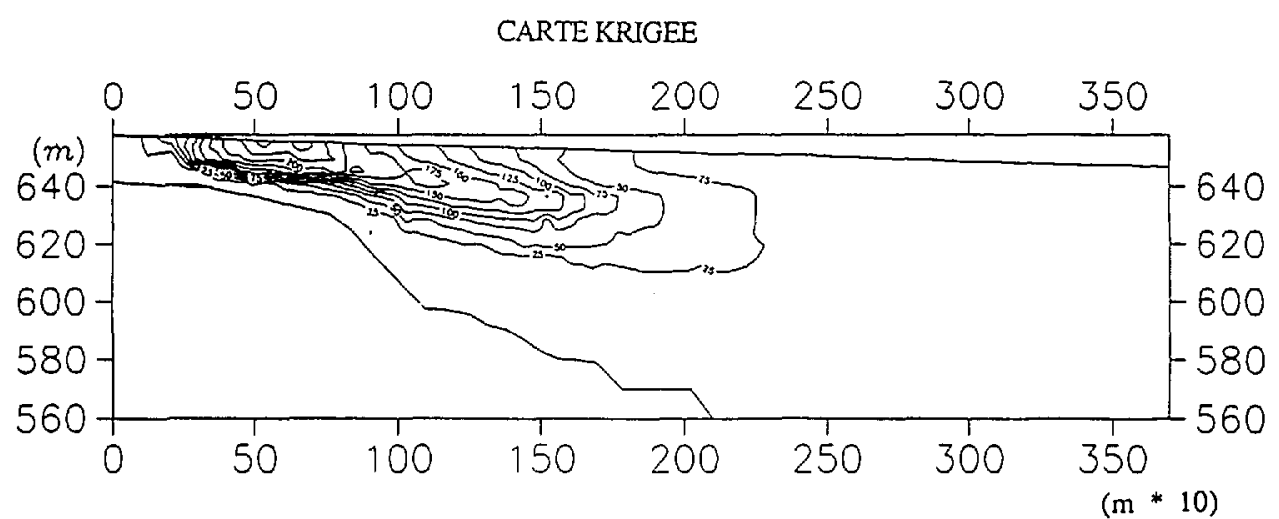

Fig. 4. Carte de la répartition initiale des chlorures ( $m g / l$ )

Im Rahmen einer regionalen Grundwasseruntersuchung musste aber 1978 festgestellt werden, dass Schmutzstoffe aus den Ablagerungsprodukten ins Grundwasser gelangten.

\subsection{Hydrogeologische Bedingungen des Grundwassers}

Die hydrogeologische Situation in der Umgebung der Deponie kann kurz wie folgt beschrieben werden (Fig. 2). Die Oberfläche des Grundwassers in den Lokkergesteinen der Talfüllung liegt relativ nahe der Terrainoberfläche. Je nach der geologischen Abfolge und trotz ihres relativ heterogenen Charakters können wir zwei sich überlagernde Grundwasserleiter unterscheiden, welche von drei Horizonten mit geringerer Durchlässigkeit begrenzt sind.

Das Grundwasserniveau schwankt im Laufe des Jahres periodisch, wobei es an den talabwärts liegenden etwa $2 / 3$ der Deponielänge bis in die Deponiesohle eindringt.

Beim maximalen Hochwasserstand wird bis $\sim 60000 \mathrm{~m}^{3}$ des Deponiematerials durch Grundwasser benetzt.

Bei Niedrigwasserbedingungen (Fig.3) drainiert die Rhone in den oberen Schichten das Grundwasser linksufrig und speist das Grundwasser rechtsufrig. Das Grundwasser der tiefer liegenden Schotterschichten fliesst von der linken Rhoneseite zur rechten Seite unter der Rhone durch.

Bei Hochwasser (Fig. 3) infiltriert die Rhone linksufrig im oberen Teil der Deponie, zusätzlich fliessen grössere Grundwassermengen vom Gamsaschuttkegel her, es resultiert ein Fliessgradient längsweg zur Deponie und weitgehend parallel zur Rhone. Im unteren Teil der Deponie beginnt dann wieder die Drainage durch die Rhone.

\subsection{Verschmutzungsverfahren}

Das Verschmutzungsverfahren kann also mit dem folgenden Satz zusammengefasst werden: Die durch Meteorwasser eluierten Substanzen reichern sich an der Deponiesohle an, werden durch die saisonalen bis in die Deponiesohle reichenden, vertikalen Grundwasserschübe ausgelaugt und durch die tieferliegenden, horizontalen Grundwasserströme weiterverfrachtet.

\section{Grundwassersanierung bzw. \\ Grundwasserschutz}

\subsection{Alternativen}

Folgende Alternativen wurden überprüft:

- Verschmutztes Grundwasser abpumpen und in den Rotten (Rhone) ableiten

- Verschmutztes Grundwasser abpumpen, reinigen und in den Rotten oder ins Grundwasser ableiten bzw. zurückpumpen

- Umschichtung des gesamten Deponiematerials auf eine dichte Deponie $(\sim 2$ Mio. $\mathrm{m}^{3}$ )

- Unterirdisches Abdichten der Deponiesohle auf dem gesamten Umfang

Daraufhin wurde die Ablagerung von verschmutzten Rückständen sofort eingestellt und Untersuchungen über Mechanismus und Ausmass der Stoffverfrachtung ins Grundwasser eingeleitet.
- Abpumpen und dadurch Herabsetzen des Pegelstandes unterhalb der Deponie

- Abdichten der Deponiefläche 
$(\mathrm{mg} / \mathrm{l})$

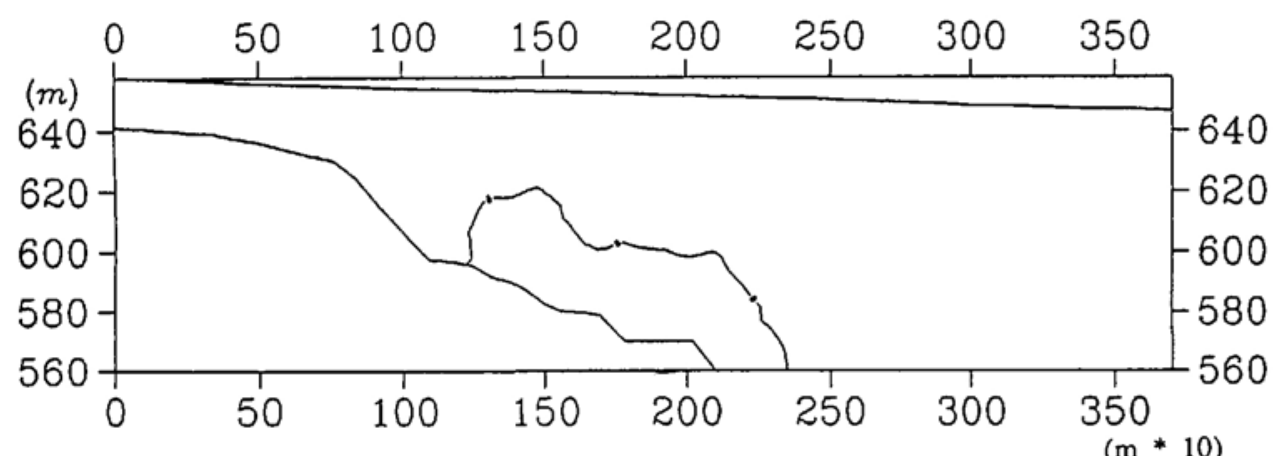

Fig. 5. Etat de la répartition des chlorures après 2,5 ans. Aucun polluant n'arrive de la décharge.

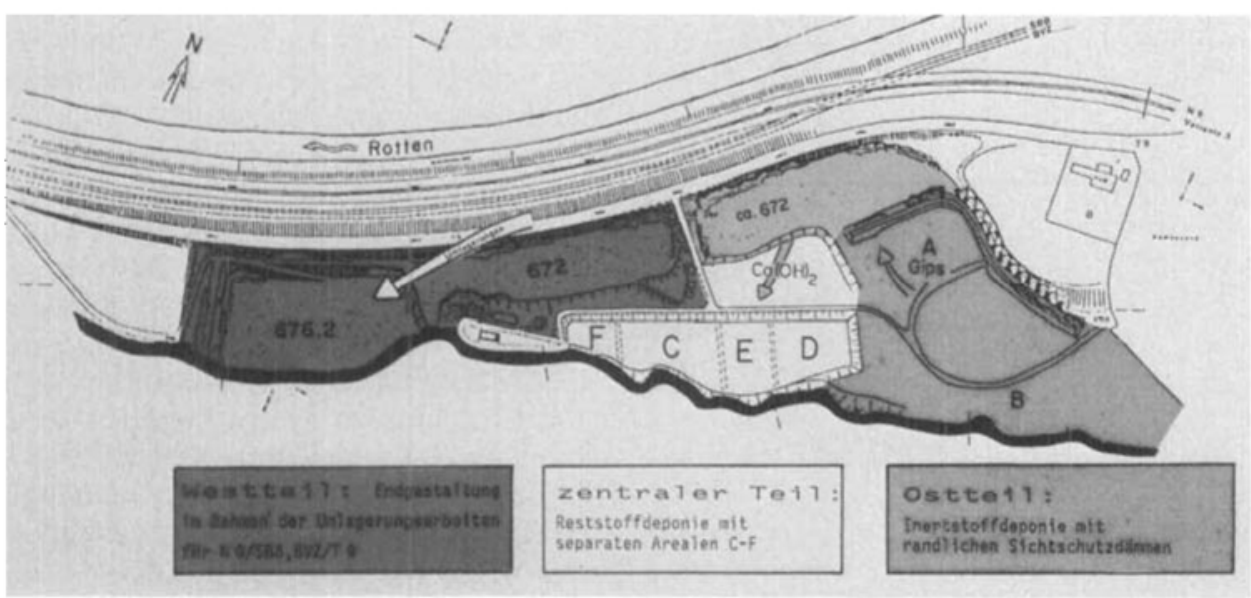

Fig. 6. Deponie Gamsenried, Lonza

Alle diese Alternativen wurden aus verschiedenen Gründen wie unverhältnismässig hohe Kosten, zu grosse Wassermenge oder definitive Schliessung der Deponie nicht weiterverfolgt.

\subsection{Aufgabe der ausgewählten Sanierung} und deren technische Beschreibung

Im Bereich der Deponie ist die Strömungsrichtung der obersten Grundwasserschicht so auszurichten, dass kein Wasser aus dem Deponiebereich wegfliessen kann.

Damit diese Umkehr der Strömungsrichtung ohne allzugrosse Senkung des Grundwasserspiegels im Bereich der Deponie erreicht werden kann, müssen auf der Zustromseite (Ostrand) spezielle Massnahmen getroffen werden. Das Konzept sieht vor, im Zustrombereich eine Absenkung des Grundwasserspiegels durch Förderung von sauberem Grundwasser zu erreichen. Vgl. dazu Fig. I. Dazu werden sechs grosse Vertikalfilterbrunnen östlich der Deponie installiert. Bei Hochwasser ist mit einer Wassermenge von rund $490 \mathrm{~m}^{3} / \mathrm{h}$ zu rechnen. Diese grossen Filterbrunnen müssen also folgende, teilweise gegensätzliche Zielsetzungen erfüllen:

- Die Absenkung muss stark genug sein, um den Gradienten des Grundwasserspiegels unter der Deponie genügend herabzusetzen und in den oberen
Grundwasserschichten eine Barrierenwirkung gegenüber dem zufliessenden Grundwasser zu erreichen.

- Das geförderte Grundwasser darf nicht verschmutzt sein, damit eine direkte Ableitung in den Rotten zulässig ist. Der untere Kulminationspunkt der Absenkung muss somit noch ausserhalb der verschmutzten Zone unter der Deponie liegen.

- Weder im Deponiebereich, noch in den Landwirtschaftsgebieten nördlich des Rottens dürfen die natürlichen Grundwasserspiegelschwankungen durch die Wasserentnahme massgeblich beeinflusst werden.

Auch die Brunnen auf dem Areal der Deponie, aus denen das verschmutzte Grundwasser gefördert wird, müssen hinsichtlich ihrer Lage und Auslegung sorgfältig an die bestehenden Verhältnisse und an die technischen Anforderungen angepasst werden:

- Einerseits muss ihre Förderleistung genügend sein, um die Strömungsrichtung der obersten Grundwasserschicht innerhalb des projektierten Einflussbereiches gegen die Deponie zu richten.

- Andererseits darf durch die Förderung von verschmutztem Grundwasser keine zusätzliche Infiltration vom Flussbett der Rhone aus hervorgerufen werden.
Aus den 12 Brunnen im Deponieareal werden rund $31 \mathrm{~m}^{3} / \mathrm{h}$ verschmutztes Grundwasser gefördert. Dieses Wasser wird ins Werk Visp weitergepumpt und dort behandelt.

Im Herbst 1990 wird die Grundwassersanierung in Betrieb sein, und ihre Auswirkung wurde dank einem mathematischen Simulieren des zweidimensionalen Schmutzstofftransportes vorgerechnet: Zum Beispiel könnte die Chlorid-Konzentration, eine typische Leitsubstanz, bei einem Sanierungserfolg von $100 \%$ nach 27 Monaten den natürlichen Werten entsprechen (Fig. 4 und 5).

\section{Anpassung der Deponie}

\subsection{Zuordnung der Deponieareale}

Aufgrund der Übersicht (Tab. 1) über die zu erwartenden Abfallkategorien und der spezifischen Randbedingungen für jede Stoffgruppe wurde eine Gliederung des Deponieareals vorgenommen: Nach heutiger Schätzung wird der neugeschaffene Deponieraum (Fig. 6) für die nächsten 30 Jahre ausreichen.

\subsection{Reststoffdeponie}

Die Areale C, D, E und F bilden die Reststoffdeponie und können mit den folgenden Zahlen charakterisiert werden (Tab. 2).

\subsection{Bauliche Massnahmen}

\subsubsection{Basisabdichtung}

Die Bereitstellung von Deponiefeldern für die zukünftige, ordnungsgemässe Ablagerung von Reststoffen erfordert den Bau einer dauerhaften Basisabdichtung sowie eines wirksamen Entwässerungssystems. Damit die Funktionstüchtigkeit dieser technischen Systeme unter der wachsenden

Tab. 1. Gliederung des Deponieareals

\begin{tabular}{|c|c|c|}
\hline Areal & Material & Abfall $\mathrm{m}^{3} / \mathrm{a}$ \\
\hline$A$ & $\begin{array}{l}\text { Deponie für Gips aus der Am- } \\
\text { moniak-Rückgewinnung }\end{array}$ & $8000-12000$ \\
\hline B & $\begin{array}{l}\text { Deponie für sauberen Bau- } \\
\text { schutt und übriges Inertmate- } \\
\text { rial }\end{array}$ & $3000-6000$ \\
\hline C & $\begin{array}{l}\text { Deponie für Schlacke aus der } \\
\text { Kehrichtverbrennung }\end{array}$ & 4500 \\
\hline D & $\begin{array}{l}\text { Deponie für Asche aus der } \\
\text { Schlammverbrennung der } \\
\text { ARA }\end{array}$ & $3000-5000$ \\
\hline E & $\begin{array}{l}\text { Deponic für inertisierte Rück- } \\
\text { stände der Rauchgasreinigung } \\
\text { von der Kehrichtverbrennung }\end{array}$ & 3500 \\
\hline $\mathrm{F}$ & $\begin{array}{l}\text { Deponie für übrige zugelas- } \\
\text { sene Reststoffe }\end{array}$ & $80-200$ \\
\hline
\end{tabular}

Tab. 2. Reststoffdeponie

\begin{tabular}{lll}
\hline Areal & $\begin{array}{l}\text { Grundfläche } \\
{\left[\mathrm{m}^{2}\right]}\end{array}$ & $\begin{array}{l}\text { Nettovolumen } \\
{\left[\mathrm{m}^{3}\right]}\end{array}$ \\
\hline C & 10700 & 130000 \\
D & 8600 & 117500 \\
E & 6700 & 100000 \\
F & 4000 & 40500 \\
Total & 3 ha & 388000 \\
\hline
\end{tabular}




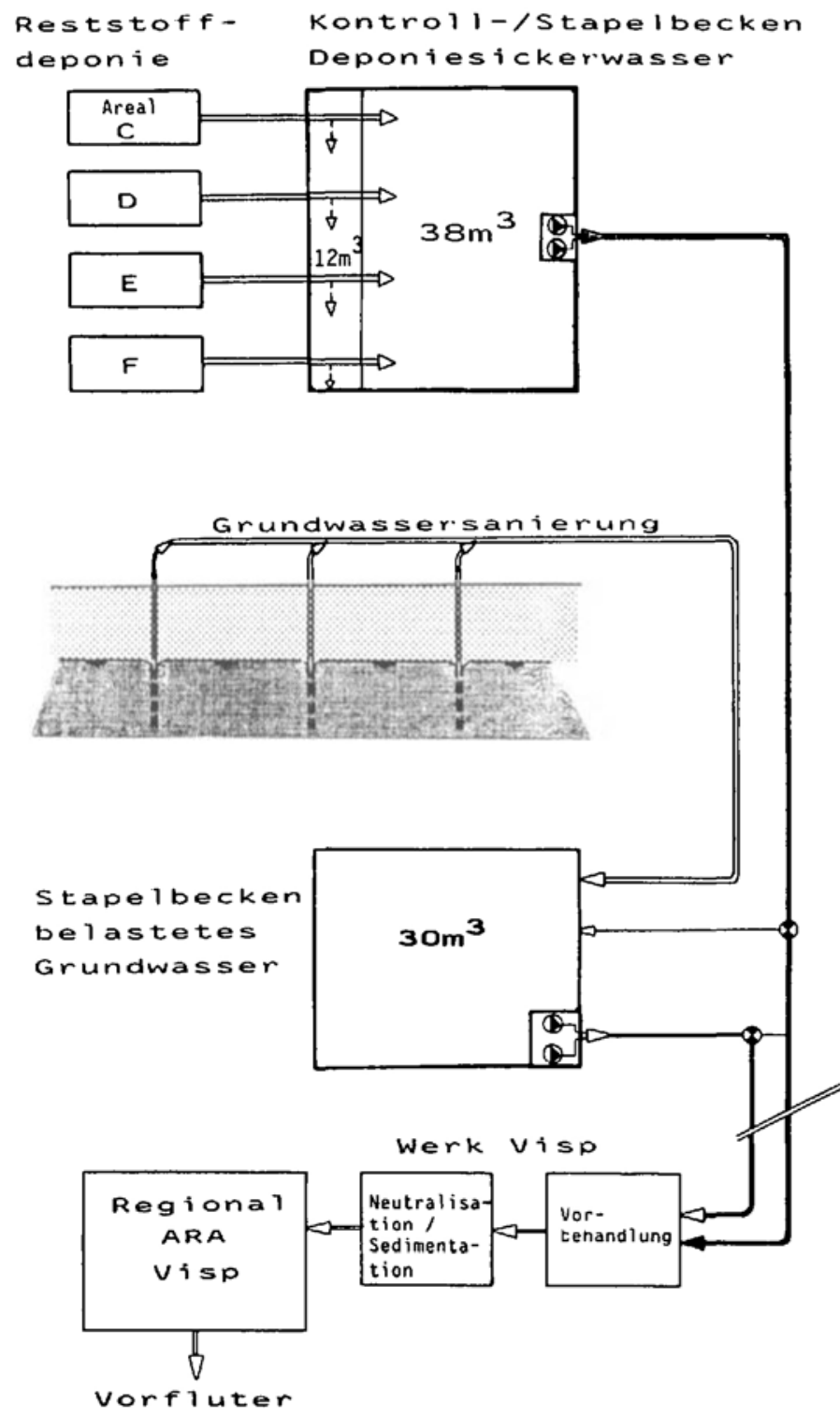

Fig. 7. Sickerwassersammlung und -entsorgung

Deponieauflast nicht beeinträchtigt wird, muss die Basisabdichtung auf einem tragfähigen Untergrund aufliegen, und zwar wo die natürliche Talsohle nur noch eine geringe Überdeckung mit Deponiematerialien aufweist.

Der zeitweise hohe Grundwasserspiegel lässt es aber auch nicht zu, dass die Basisabdichtung direkt auf dem tragfähigen Untergrund aufgebaut wird. Die Sohle muss daher mit schichtweise eingebrachtem und verdichtetem Inertmaterial soweit angehoben werden, dass sie auch bei einem extremen Hochwasser trockenliegt.

Nach dem heutigen Stand der Deponietechnik stehen die drei folgenden Systeme zur Verfügung:

- mineralische Abdichtung (Grundmaterial mit Betonitzusatz),

- Kunststoff-Dichtungsbahnen (mit mineralischer Trag- und Schutzschicht),

- bituminöser Dichtungsbelag (evtl. mit mineralischer Schutzschicht).
Alle diese Möglichkeiten erfüllen vom technischen Standard her die Anforderungen. Die Wahl des bestgeeigneten Systems wird erst im Rahmen der Submission und der Detailprojektierung möglich sein.

\subsubsection{Randabdichtung}

Die Deponieareale grenzen im Süden an die steil aufsteigende, felsige Talflanke mit einer sehr unregelmässigen Oberfläche. Die Abdichtung dieses Bereiches ist nur etappenweise von der jeweiligen Deponieoberfläche her möglich. Damit reduziert sich die Auswahl der Abdichtungssysteme auf Dämme mit mineralischem Abdichtungsmaterial, da alle übrigen Verfahren mit grossen, technischen Schwierigkeiten und einem unverhältnismässigen Aufwand verbunden wären.

Die mineralischen Abdichtungsdämme werden je nach Böschungsneigung und Materialeigenschaften in einer Höhe von 1-2 m etappenweise aufgebaut. Entlang der südlichen Felsflanke wird zur Ableitung des auf der Felsoberfläche zufliessenden Wassers ein minimal $1 \mathrm{~m}$ starker Sikkerkörper hinter den Dichtungsdämmen eingebaut.

Die randlichen Dichtungsdämme werden analog zur mineralischen Basisabdichtung in Einzelschichten von $20-30 \mathrm{~cm}$ aufgebaut und sorgfältig verdichtet.

\subsubsection{Sickerwassersammlung und -entsorgung (Fig. 7)}

Das Sickerwasser der Deponien C, D, E und $F$ wird in separaten Leitungen zum Kontroll- und Stapelbecken geführt. Das Becken bietet Platz für ein Stapelvolumen von total $c a .50 \mathrm{~m}^{3}$. Dieses wurde in ein grösseres Becken von rund $38 \mathrm{~m}^{3}$ Inhalt und in ein kleineres Separatbecken mit einem Volumen von $\mathrm{ca} .12 \mathrm{~m}^{3}$ unterteilt. Diese Aufteilung des Beckens verschafft die Möglichkeit, Sickerwasser aus einzelnen Deponiearealen separat zu sammeln und bei Bedarf einer speziellen Behandlung (z. B. Elimination einzelner Inhaltsstoffe) zuzuführen.

Die getrennt geführten Leitungen der Deponien C, D, E und F münden einzeln in ein kleines Einlaufbecken, das eine manuelle Mengenmessung und eine getrennte Probenahme erlaubt. Normalerweise fliesst das Sickerwasser von dort ins grössere Sammelbecken, doch kann der Einlauf jeder Deponie durch eine einfache Überleitung ins kleinere Sammelbecken abgetrennt werden.

Diese Aufteilung bietet eine optimale Flexibilität bezüglich der anschliessenden Sickerwasserreinigung, indem die zur Hauptsache nur schwach belasteten Sikkerwässer von allfällig stärker verschmutzten Zuflüssen abgetrennt werden können.

Ab dem Stapelbecken wird das Sickerwasser über eine Druckleitung zur Vorbehandlung ins Werk Visp gepumpt und anschliessend in der ARA gereinigt.

\subsection{Finanzieller Aufwand}

Die Investitionskosten betragen insgesamt 32,5 Mio. Fr., davon 19,0 Mio. für die Lonza $A G$, Werk Visp, und 13,5 Mio. für den Gemeindeverband Oberwallis für die Kehrichtbeseitigung (GVO).

\subsection{Inbetriebnahme}

Die Inbetriebnahme der Reststoffdeponie ist für Mitte 1992 vorgesehen.

\section{Regionales Entsorgungskonzept}

Unter Einbezug aller Entsorgungsanlagen der Lonza $A G$ in Gamsen (Deponie) und in Visp (RVA, ARA) sowie der Kehrichtentsorgung des Gemeindeverbandes wird ein regionales Entsorgungskonzept im Interesse der Arbeitsplatzsicherung und der Öffentlichkeit verwirklicht. 